\title{
A Stiff Tendon Neuromusculoskeletal Model of the Knee
}

\author{
Massimo Sartori, David G. Lloyd, Monica Reggiani, and Enrico Pagello
}

\begin{abstract}
Now more than ever, progresses in information technology applied to rehabilitation robotics give new hopes to people recovering from different kinds of diseases and injuries. Beside the standard application of EMG signals to analyze disabilities or to track progress in rehabilitation, more focus has been put on controlling robot arms and exoskeletons. In recent years, biomechanists have developed very complex neuromusculoskeletal (NM) models of human joints to understand how the nervous system controls muscles and generates movements. Aware of these potentials, we have started a process of simplification to obtain a NM model suitable for the realtime control for a lower extremity exoskeleton. In this paper we present the NM model for the knee previously developed by Lloyd et al. [1]. We then investigate the effects of assuming the tendon infinitely stiff and show how this simplification does not affect the capacity of the model to predict muscle force and joint moment. We also assess the decrease in processing time required to calibrate the model and perform runtime estimates of muscles. Finally, we illustrate the implications of our research for the health care economic and social systems.
\end{abstract}

\section{INTRODUCTION}

The significant aging of population, with the associated increase in injuries and disabilities, calls for new solutions to health care. Elderly should be able to stay at home longer, thus reducing the cost of elderly care. People with disabilities, whose numbers are on the rise, should be able to continue to work to maintain the workforce productivity.

Mobility represents a basic need that has to be guaranteed to ensure independence and to boost the integration of disable people in the society. An effective way to strength and restore mobility and to improve coordination is the musculoskeletal therapy. Despite its effectiveness, access to these treatments is still limited because they are quite expensive as they continuously require the knowledge and skills of several trained therapists.

Robotics rehabilitation promises to be a cost-effective solution. With the support of a robotic exoskeleton, for instance, the therapist is relieved from the duty of constantly manipulating the patient's limbs throughout the rehabilitation treatment. Furthermore, on board sensors can also acquire accurate kinematics and biophysical data and provide meaningful information on current patient's state. Their elaboration can help therapists and doctors in tracking patient's progress. Finally, rehabilitation robots enable the possibility

M. Sartori and E. Pagello are with the Department of Information Engineering, University of Padova and with the Institute of Biomedical Engineering - National Research Council, Padova, Italy. massimo.sartoriddei.unipd.it,

D. G. Lloyd is with the School of Sport Science Exercise and Health, University of Western Australia,

M. Reggiani is with the Department of Technique and Management, University of Padova, Vicenza, Italy. to develop telerehabilitation applications that could allow doctors to remotely supervise patients training at home. The obvious importance of this research domain has also been highlighted in two recent documents drawing the future of robotics research in Europe [2] and in the United States [3].

Rehabilitation robotics offers several challenges. Key ones are the development of intuitive human-robot interfaces (HRI), supporting the interaction of robotics devices with the subject, and the robot's ability to autonomously adapt to current patient's capabilities. The facing of these challenges would benefit from the availability of a NeuroMusculoskeletal Model (NMM). NM modeling can potentially enhances the interaction between the human and the rehabilitation device as it is a powerful tool for understanding how the nervous system controls muscles to generate movements. Both the biomechanics and robotics communities have successfully used NM model in several applications. Biomechanists have developed complex uniarticular joint models driven by surface electromyography (sEMG) signals for the purpose of studying human motor control strategies. Their complexity, however, relegate them to offline computation [1]. Only recently, robotics researchers have developed NM models suitable for real-time applications [4], [5], [6], [7]. In [4], the authors present a model of the human muscles able to predict joint torques for the upper limb as a function of the joint kinematics and neural activation level. Another effective research has been presented in [5]. The EMG signals are used as input for a simplified biomechanical model able to derive the desired action of the operator and control an orthoses for the knee joint. These models are however far too simple and do not allow complex movements in the supported limbs. The availability of accurate and comprehensive neuromusculoskeletal models, combining high reliability and realtime operation, is therefore needed for the development of effective HRI and control system for rehabilitation robotics devices like exoskeletons.

This paper presents an EMG-driven neuromusculoskeletal model for the knee joint. We focused on the human leg due to the lack of research when compared with the advancements on upper extremity exoskeletons. The starting point for this research is the complex NM model previously developed by Lloyd et al. [1]. With the goal of reducing time requirements for real-time applications while retaining the required reliability, we have investigated the impact of assuming the tendon infinitely stiff. Experimental results demonstrate that this assumption does not affect the capacity of the Stiff Tendon Model (STM) to predict muscle forces and joint moments during gait. We also assessed a significant reduction in processing time for the calibration and the 


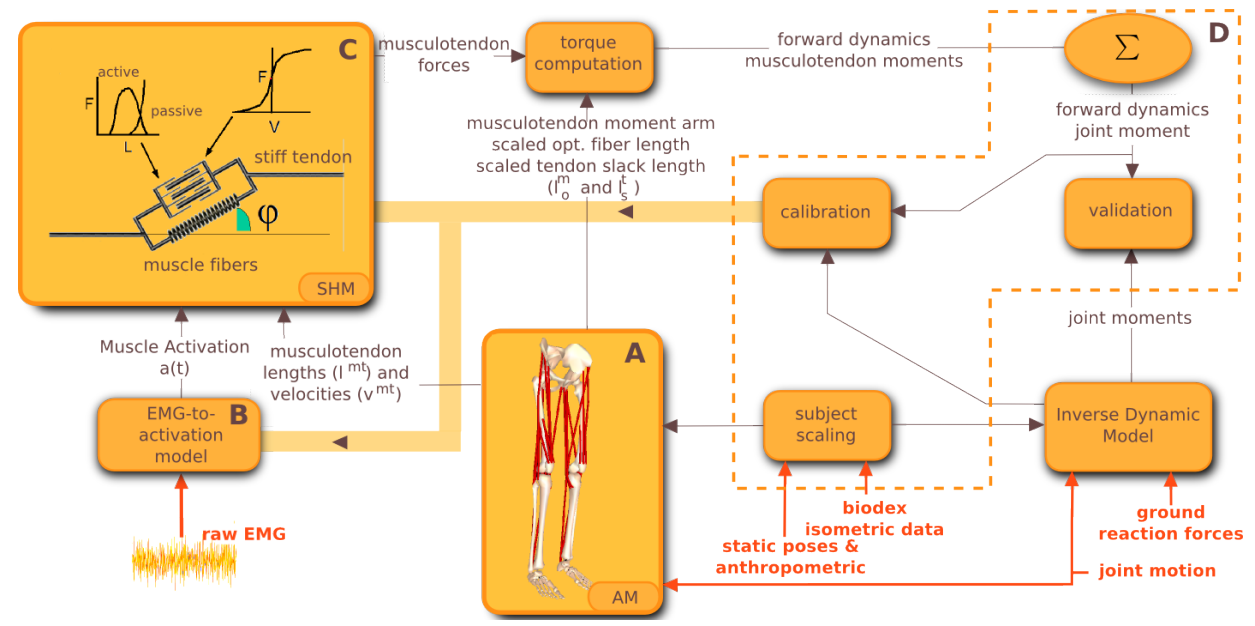

Fig. 1. Schematic of the EMG-driven stiff tendon model. Static poses together with anthropometric parameters and biodex data are used to scale the AM (block A) and estimate the following terms: $l^{m t}, v^{m t}, l_{0}^{m}, l_{s}^{t}$ and MTU moment arms. Raw EMGs are converted in muscle activation (block B) and are subsequently fed into the SHM (block C) together with the outputs provided by the AM. The SHM, in turn, computes musculotendon forces that are combined with their respective musculotendon moment arms and summed up together to produce an estimate on the knee joint moment. This estimate is used, in block D either for validating the model or to calibrate the remaining model parameters.

runtime estimation of muscle forces and joint moments.

The remaining of the paper is organized as follow. Sec. II presents the general architecture of the STM. Sec. III describes the theoretical backgrounds behind the design of the model assuming the tendon stiff. A section with the details about the data acquisition procedure follows (Sec. IV) while results supporting our research are presented in Sec. V. Finally, Sec. VI discusses the socioeconomic impact of our research.

\section{MODEL STRUCTURE}

In this section we briefly review the NM model developed by Lloyd et al. and presented in [1], [8], [9], [10]. The EMGdriven neuromusculoskeletal (NM) model (Fig. 1) consists of 4 fundamental components: anatomical model (Fig. 1A), EMG-to-activation model (Fig. 1-B), stiff tendon Hilltype muscle model (Fig. 1-C), and scaling, calibration and validation of the NM model (Fig. 1-D). The NM model uses both forward and inverse dynamics in an hybrid approach to EMG-driven modeling. The only inputs are raw EMG and joint kinematics. In Sec. II-C we illustrate the main contribution of this paper, i.e. the changes we applied to the Hill-type muscle model to decrease processing time. Part of the Sec. II-D discusses the extension introduced in the subject scaling model.

\section{A. Anatomical Model}

The anatomical model (AM) of the lower limb (Fig. 1A) is created using the SIMM Biomechanics Software Suite (Musculographics, Inc.) based on the results presented in [11], [8]. The AM consists of line segment representations of 13 musculotendon units (MTUs) spanning the knee joint including: semimembranosus, semitendinosus, biceps femoris long head, biceps femoris short head, sartorius, tensor fascia latae, gracilis, vastus lateralis, vastus medialis, vastus intermedius, rectus femoris, medial gastrocnemius, and lateral gastrocnemius. Only two muscles crossing the knee are not included: the plantaris and the popliteus. They are assumed to have a negligible contribution to the total flexion-extension (FE) moment due to their relatively small physiological cross sectional area. The lengths of the modeled bones and MTUs are linearly scaled to the actual subject's size. The AM is kinematic driven by the hip, $\theta_{h}$, knee, $\theta_{k}$, and ankle, $\theta_{h}$, joint angles including both uniarticular and biarticular muscles to determine muscletendon lengths, $l^{m t}$, velocities, $v^{m t}$, and moment arms, $r$.

\section{B. EMG-to-activation Model}

Raw EMG signals from each muscle are band pass filtered $(10-150 \mathrm{~Hz})$ using a second order Butterworth filter and subsequently full wave rectified and low pass filtered $(6 \mathrm{~Hz})$ to obtain the linear envelope. Envelopes are then normalized against maximum values obtained from linear envelopes computed during isometric maximal voluntary contractions (MVC). Then, the EMG-to-activation model (Fig 1-B) applies a recursive filter to obtain an estimate of the muscle activation $(u(t))$ [1]. Muscle activation is further processed to account for the non-linear relationship between EMG and muscle force. The model uses an exponential relationship that includes a single parameter to control the extent of the non-linear relationship [9]:

$$
a(u(t))=\frac{e^{A u(t)}-1}{e^{A}-1}
$$

where $A$ is the non-linear shape parameter and it is constrained to $-5<A_{i}<0$, with 0 being a linear relationship.

\section{Stiff Tendon Hill-type Muscle Model}

This section presents the main contribution of our work, i.e. the specific changes that we introduced in the NM model designed by Lloyd et al. [1]. The main modification consists in replacing in the Hill-type muscle model the non-linear 


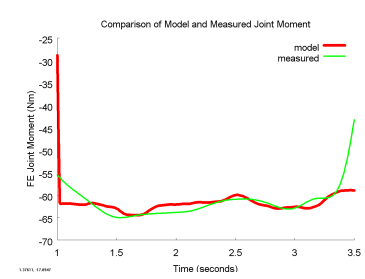

(a)

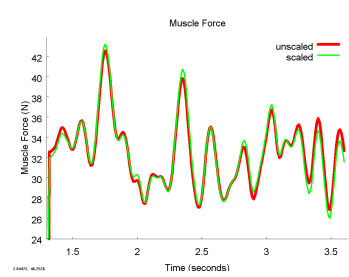

(b)

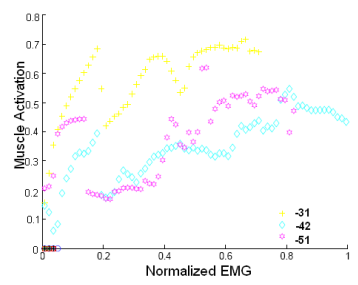

(c)

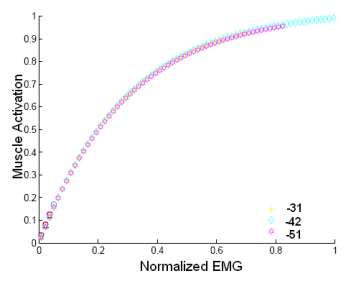

(d)

Fig. 2. (a) Recorded and estimated knee moment during an isometric trial. (b) The muscle forces predicted by the NM model are scaled to exactly reproduce the measure moment. In this graph the semimembranosus muscle forces are displayed. (b) EMG-to-activation relationship for the semimembranosus muscle before (c) and after (d) calibration (flexion isometric contraction).

tendon with a stiff one. The modified model will be referred as Stiff Tendon Hill-type muscle Model (SHM).

The output from the previous two components is the input for the SHM (Fig. 1-C) that estimates the forces generated by each muscle. This forces are then used to determine the resultant knee joint moment. The SHM represents each MTU as a contractile and stretchable element (modeling the muscle fiber) in series with a tendon. The contractile-stretchable element of the muscle fiber is modeled as a combination of general force-length and force-velocity functions (Fig. 1C). Two are the force-length functions used to compute the force generated at a defined fiber length. The active forcelength function $\left(f_{A}\left(\tilde{l}^{m}\right)\right)$ refers to the contractile element of the fiber producing active force when contracting. The passive force-length function $\left(f_{P}\left(\tilde{l}^{m}\right)\right)$ represents the passive element of the fiber producing resistive force when stretched. Both depends on $\tilde{l}^{m}$, the muscle fiber length normalized with respect to the optimal fiber length $l_{0}^{m}$. This parameter is the length at which a muscle produce the maximal force. The force-velocity function $\left(f_{V}\left(v^{m}\right)\right)$ expresses the influence of the fiber contraction velocity $\left(v^{m}\right)$ on the muscle force.

The final muscle fiber force $\left(F^{m}\right)$ depends on the following parameters: maximal isometric muscle force at optimal fiber length $\left(F_{0}^{m}\right)$, muscle strength $(\delta)$, instantaneous fiber length $\left(l^{m}\right)$ and velocity $\left(v^{m}\right)$, and, finally, instantaneous optimal fiber length $\left(l_{o}^{m}\right)$ and instantaneous pennation angle $(\varphi)$, both functions of muscle activation $(a(u))$.

The proposed NMM models the tendon as a nondeformable infinitely stiff tendon-like body, in contrast to the model previously developed by Lloyd et al. [1], that includes a non-simplified Hill-type muscle model. In the following, we will refer to this model as Lloyd Hill-type Model (LHM). The LHM models the tendon as a passive elastic element that acts like rubber bands. As the muscle fiber force grows, the tendon gets stretched. When the tendon resting length $\left(l_{s}^{t}\right)$ is exceeded, the tendon produces a resistive force as the strain increases. The tendon contribution to the musculotendon force is generally described by a non-linear function that expresses the relationship between force and instantaneous tendon strain. However, according to [12] the tendon is rather stiff: the strain is only the $3.3 \%$ of the tendon length when the muscle generates maximum isometric force. Therefore, we decided to neglect the tendon strain in our model resulting in MTU length and force being dependent only on the fiber length variation. This allows to derive an analytical formula for estimating the muscle fiber length and velocity and avoids the time-consuming Runge-Kutta-Fehlberg (RKF) integration of the very stiff muscle-tendon differential equations needed in the LHM [1]. Section III presents an in-depth description of the SHM design. Section V shows how our assumptions positively influence the prediction of muscle forces and knee joint moment.

Once the muscle forces are computed, the net knee joint flexion-extension moment is obtained by summing the product of each muscle's force by its flexion-extension moment arm (Fig. 1-D).

\section{Scaling, Calibration and Validation of the Model}

To validate a model, outputs are usually compared with data measured empirically. However, due to the methodological difficulties in measuring individual muscle forces, any direct validation of the NM model on humans is prevented. An indirect validation process was therefore used to validate the model prediction ability and also to calibrate and scale the model to each subject. The estimated knee joint FE moment is compared with the net FE moment experimentally measured with an inverse dynamic model [13]. When the model is able to accurately estimated the muscle forces, the computed knee FE moment should be equal to the measured one.

The aim of the calibration process is to define the values of a set of model parameters tuned to the subject and thus allowing the proper estimation of the FE knee moment. Starting from a set of calibration trials, a simulated annealing process alters the selected parameters to minimize the sum of squared differences between the net knee FE moment approximated by the model and that experimentally measured (Fig. 1-D) [1]. The adjustable parameters used in the calibration process are divided in two main groups. The first group includes parameters of the EMG-to-activation model such as the activation variables $C_{1}$ and $C_{2}$ that are used by the recursive filter to determine $u(t)$ (Sec. II-B) and the non linear shape factor $A$ (Eq. 1). These parameters account for changes in muscle excitation level due to EMG 
electrodes placement, skin preparation and impedance and need to be calibrated in each testing session. The second group includes parameters of the Hill-type muscle model: flexor $\left(\delta_{f}\right)$, extensor $\left(\delta_{e}\right)$ and gastrocnemius $\left(\delta_{g}\right)$ strength coefficients. These parameters scale the relative $F_{0}^{m}$ values to account for individual differences in muscle strength. They also need to be calibrated each session.

There are also additional parameters that are required to be calibrated only once for each subject as they depends only on the body geometry. These parameters take into account the musculoskeletal geometry of the subject ("subject scaling" block in Fig. 1-D). They include the tendon slack length $\left(l_{s}^{t}\right)$ and the optimal muscle fiber length $\left(l_{0}^{m}\right)$, the length at which the muscle produces maximal force. They both have great influence on the force generating behavior of a MTU and they not-linearly change with the subject dimension. The scaling of these parameters is performed according to the method described in [10].

Since the model presented in this paper assumes the stiffness of the tendon, then the scaling of $l_{s}^{t}$, the tendon slack length, deserves even more attention. A technique to refine the previously scaled $l_{s}^{t}$ values has been presented by the authors in [14]. This technique preserves the nonlinear EMG-to-activation relationship expressed in Eq. 1 for an individual muscle over several isometric trials at different joint angles.

Muscle activation is rearranged from Eq. 3 to express its relation with muscle fiber length as follows:

$$
a_{u}=\frac{\frac{F^{m}}{F_{0}^{m} \delta}-f_{P}\left(\tilde{l}_{i}^{m}\right)}{f_{A}\left(\tilde{l}^{m}\right)}
$$

where the contraction velocity has been neglected since the tasks have been performed under isometric conditions. The tendon slack length (influencing $\tilde{l}_{i}^{m}$ ) is refined to minimize the discrepancy among the EMG-to-activation relationships over different trials (Fig. 2-c). The term $F^{m}$ in Eq. 2 is an estimate of the individual muscle force. This estimate has been derived using the NM model calibrated on each isometric trial. When deriving muscle forces using the NM model, a perfect match between the recorded net knee moment and the estimated one cannot be achieved (Fig. 2a). This happens because, during the calibration process, the tendon slack length has not been properly scaled yet and a small number of parameters are varied. We therefore scaled each muscle force by the term: $T_{R} / T_{E}$, with $T_{R}$ the knee reference torque and $T_{E}$ the estimated one. This allows to obtain force estimates that generate the actual reference joint torque and preserve the force sharing predicted by the NM model (Fig. 2-b). Forces were scaled so that the corresponding activation level was always between 0 and 1. Experimental results in Fig. 2-c shows a significant discrepancy between the EMG-to-activation relationships over different angles before the scaling of the tendon slack length. Fig. 2-d shows the same relationship after calibration. The EMG-to-activation relationship is now preserved over all trials.

\section{DESIGN OF THE SHM}

This section provides additional details about the design of the Stiff tendon Hill-type muscle Model (Fig. 3). As shown in Sec. II-C, the SHM computes the muscle fiber force combining together the action of the active and passive elements of the fiber. The final force value is:

$$
F^{m}=\left(f_{A}\left(\tilde{l}^{m}\right) \cdot f_{V}\left(v^{m}\right) \cdot a(u)+f_{P}\left(\tilde{l}^{m}\right)+d^{m} \cdot \tilde{v}^{m}\right) \cdot F_{0}^{m} \cdot \delta
$$

where the term $d^{m}$ represents a passive parallel damping element that was added to the force-velocity relationship to account for the muscles damping characteristics as suggested in [15]. Including passive damping has the benefit of increasing the stability. Other terms of Eq. 3 have been already defined in Sec. II-C. The final force produced by each MTU can be derived considering the influence of the pennation angle $(\varphi)$, the angle at which the fibers are oriented with respect to the tendon (Fig. 1-C). The component of the muscle fiber force computed from Eq. 3 along the tendon line of action is therefore: $F^{m t}=F^{m} \cdot \cos (\varphi)$. To compute $F^{m}$ and therefore $F^{m t}$, the SHM needs to first obtain the instantaneous values for $l^{m}$ and $v^{m}$.

The method used to calculate these values is the main difference between the SHM and LHM. The Stiff tendon Hill-type muscle model is designed considering the tendon infinitely stiff and not producing any resistive force to stretch. Its length is constant and it is set to $l_{s}^{t}$. This assumption allows to easily solve the following equation for the muscle fiber length:

$$
l^{m}=\sqrt{\left(l_{0}^{m} \cdot \sin \left(\varphi_{0}\right)\right)^{2}+\left(l^{m t}-l^{t}\right)^{2}}
$$

where $\varphi_{0}$ is the pennation angle at optimal fiber length. If the tendon length $l^{t}$ is unknown, this equation could not be solved. However, since we assumed $l^{t}=l_{s}^{t}$, the equation allows to obtain $l^{m}$ with low computation time requirements. Furthermore, since the tendon is assumed to be infinitely stiff, the fiber contraction velocity equals the MTU contraction velocity. Therefore, $v^{m}$ can be computed

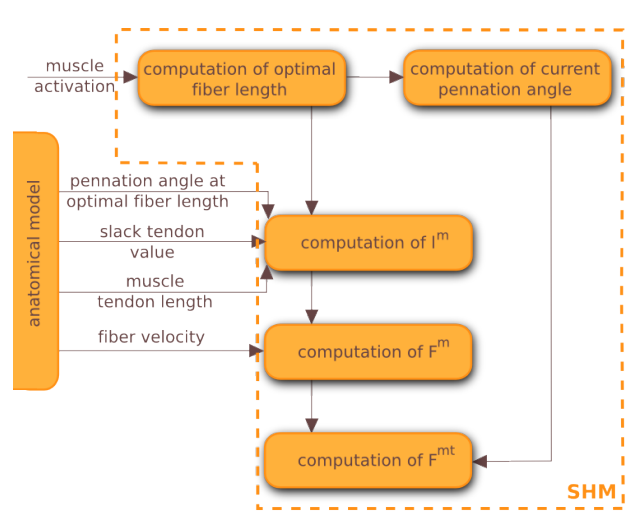

Fig. 3. Schematic of the stiff tendon Hill-type muscle model. The SHM takes inputs from the AM model and from the EMG-to-activation model. Since the tendon is stiff, $v^{m}=v^{m t}$ and can be computed by the AM. This cannot be done by LTH where $v^{m}$ has to be computed at run time by the Hill-type muscle model. 
by the AM by differentiating $l^{m t}$. This enables to speed up the run-time execution of the SHM.

Eq. 4 cannot be solved by the LHM as both $l^{m}$ and $l^{t}$ are unknown. In LHM, an initial guess on muscle fiber velocities is obtained by determining the stiffness of muscle fiber and tendon, and apportioning the total musculotendon velocity to the muscle fiber and tendon based on their relative stiffness. This is accomplished using the Loan Dynamic Force Estimation Method (LDFEM) [16]. Muscle fiber length at the following time step is then predicted by forward integration of the fiber velocity using the RKF algorithm. The instantaneous tendon length and strain can then be computed and the resultant force generated on the tendon is estimated. From this, the active and passive component of the muscle fiber force are derived and a new estimate of fiber velocity is obtained by interpolating the force velocity curve. The process can now be iterated for all time steps.

The LHM requires a longer computation time for the estimation of $l^{m}$ and $v^{m}$ when compared to the SHM as it requires the execution of the LDFEM to get the integration process started. Additionally, at each integration step, the time-consuming RKF algorithm has to be executed. The SHM, instead, only needs to solve Eq. 4 to derive $l^{m}$ while $v^{m}$ is computed by the AM. Section V shows a comparison of the performance of the two models.

\section{EXPERIMENTAL PROCEDURES}

Experiments were performed at the Gait Laboratory of the School of Sport Science Exercise and Health of the University of Western Australia. Tests involved capturing anatomical static poses of a 44 years old healthy male subject using a 7 camera motion capture system (Vicon, Oxford, UK) and then modeled to estimate individual bone lengths. This data was then used to scale the anatomical model (Sec. IIA). Optimal fiber length and tendon slack length of all MTUs were also scaled to fit subject's geometry (Sec. II-D). EMG signals were collected from the selected muscles using double-differential surface electrodes. A telemetered system (Noraxon, Scottsdale, USA) was used to transfer the EMG signals to a 16 channel amplifier (Delsys, Boston, USA) with sampling frequency at $2 k H z$. EMG were collected during two types of trials: dynamometer and gait trials. Dynamometer trials were performed using a dynamometer (Biodex, New York, USA) under isometric conditions. The knee was secured at different angles to span the whole operating range of the uniarticular muscles. Furthermore, hip and ankle joint angle positions were also varied during additional knee $\mathrm{FE}$ isometric trials to account for the action of biarticular muscles. Gait trials included the acquisition of ground reaction forces and joints kinematics, as well. All motion data was filtered using a quintic spline function with a mean square error value of 20 . Ground reaction forces data was filtered using a two-way low pass Butterworth filter with a cutoff frequency of $6 \mathrm{~Hz}$. Refer to Sec. II-B for the EMG signal processing phase. Different sets of data were then created. The first was used to further calibrate the stiff tendon NM model (Sec. II-D) and it included the dynamometer trials
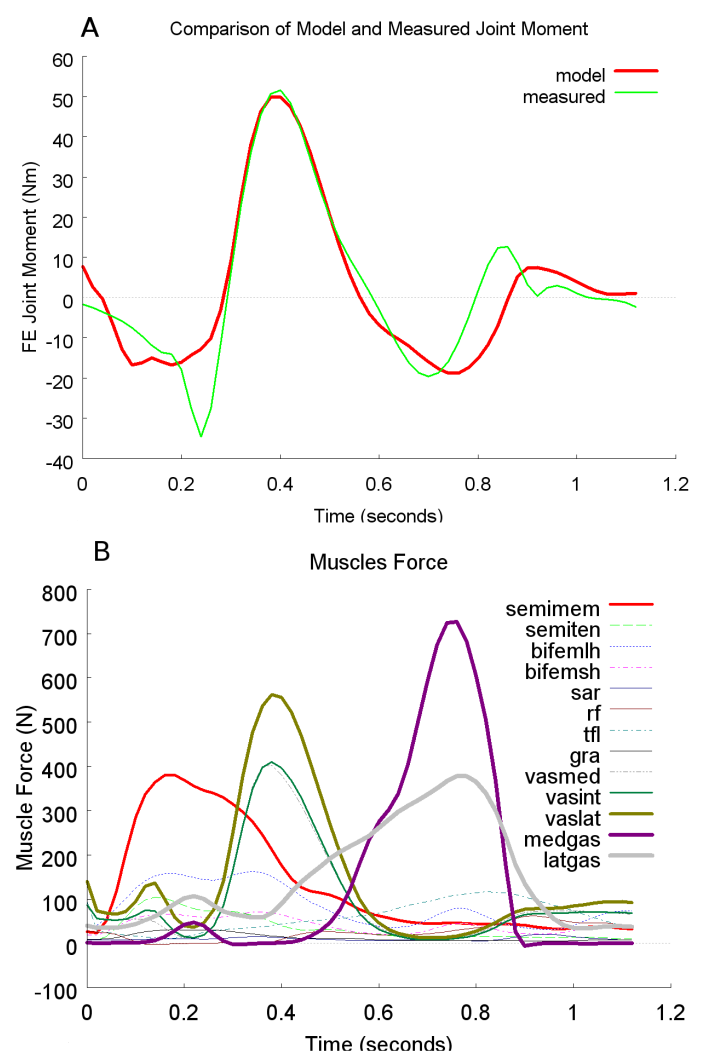

Fig. 4. (a) Model's ability to predict FE knee moment during gait trial number 5. Positive joint moments correspond to knee flexion. The crosscorrelation between estimated and measured moment was 0.876 in this particular trial with a RMSE of $8.563 \mathrm{Nm}$. Parameters of the model were adjusted using data from different trials, and then the model was used predict the moments in this gait trial. This demonstrates the model's ability to predict moments from a novel task using only EMG and kinematic information despite the tendon was assumed infinitely stiff. (b) The force contribution of 13 MTUs to the total joint moment.

and some gait trials, as well. The second data set comprised gait trials used for model validation purposes only (Sec. V).

\section{EXPERIMENTAL RESULTS}

After calibration, the stiff tendon NM model was used to predict knee moments during 10 novel gait trials with a duration of $1.2 \pm 0.06 \mathrm{~s}$. Despite assuming the tendon infinitely stiff, the prediction ability of the model was not affected. Over all trials, the level of cross-correlation $(R)$ between the estimated knee moment and the experimentally measured one was $0.892 \pm 0.047$ (with 1 meaning maximal correlation). This indicates that over all trials, our model estimates moments with proper accuracy when compared to the measured ones. The root mean squared error (RMSE) over all trials was $8.1 \pm 1.02 \mathrm{Nm}$. In the considered gait trials, experimentally measured moments values ranged from a minimum of $-33.40 \pm 3.99 \mathrm{Nm}$ to a maximum of $46.50 \pm$ $10 \mathrm{Nm}$. Therefore, the average error introduced by our model was about a $10 \%$ of the range of variation observed on the knee FE moment. A significant improvement in computation time was also achieved. The stiff tendon model took $0.0639 \pm$ $0.0015 \mathrm{~s}$ to estimates the joint moments while the complete 
TABLE I

STATISTICAL COEFFICIENTS AND PERFORMANCE DATA FOR THE 10 GAIT TRIALS.

\begin{tabular}{|c||c||c||c||c||c||c||c||c||c||c|}
\hline Trial Number & 1 & 2 & 3 & 4 & 5 & 6 & 7 & 8 & 9 & 10 \\
\hline$R$ & 0.804 & 0.924 & 0.862 & 0.927 & 0.876 & 0.927 & 0.896 & 0.835 & 0.943 & 0.926 \\
\hline$R M S E(N m)$ & 7.723 & 9.873 & 8.678 & 7.414 & 8.563 & 7.537 & 8.542 & 9.377 & 6.392 & 7.702 \\
\hline SHM Execution Time $(s)$ & 0.0630 & 0.0644 & 0.0632 & 0.0620 & 0.0631 & 0.0646 & 0.0673 & 0.0647 & 0.0639 & 0.0631 \\
\hline LHM Execution Time $(s)$ & 0.670 & 0.688 & 0.692 & 0.695 & 0.697 & 0.711 & 0.682 & 0.672 & 0.692 & 0.715 \\
\hline SHM Calibratio Time $(s)$ & 64.842 & 63.152 & 63.343 & 61.553 & 62.761 & 63.941 & 64.833 & 62.455 & 63.442 & 63.343 \\
\hline LHM Calibration Time $(h$, min $)$ & 3,48 & 3,37 & 3,10 & 2,12 & 2,45 & 2,57 & 3,12 & 3,22 & 2,37 & 3,43 \\
\hline
\end{tabular}

model took $0.691 s \pm 0.0146 s$. Calibration was completed in $63.432 \pm 1.195 \mathrm{~s}$ by the simplified model. It took more than $3 h$ on the complete one. Therefore the calibration algorithm and runtime is much faster than the previous version enabling its use in real time applications. Performance tests were executed on a PC with a Pentium 4 processor $(3.20 G H z)$ and $2 G B$ RAM memory. Table I provides additional details on performance results. The estimation of the muscle forces and fiber lengths is an intermediate step in the model that leads to the computation of the knee joint moment. Fig. 4-a shows the ability of our model to predict FE knee moment. Fig. 4-b shows the estimation of muscle forces that allowed the computation of the joint moments.

\section{IMPACT INVESTIGATION}

This section introduces the impacts of our research. First, it illustrates the possible enhancements on the control of exoskeleton devices. Then, it analyzes the possible social benefits for the health care system and market.

\section{A. Enhancements on Exoskeleton Control}

Despite the recent progresses in the design and implementation of robotic exoskeletons and powered orthoses, many design challenges are still open. Limitations of current exoskeletons can be overcome by the use of NM models. Indeed, a better understanding of muscle and tendon behavior can improve actuation and control algorithms, resulting in an enhanced biomimetic limb dynamic [6]. Our stiff tendon NM model can already capture the major features of human walking and may improve understanding of musculoskeletal morphology and neural control. This capabilities help in the design of economical, stable and low-mass exoskeletons for human walking augmentation. Another limit of today exoskeletons and orthoses is the lack of an efficient information exchange between the human wearer's nervous system and the wearable device [6]. Our EMG-driven NM model provides an effective human-machine interface that can be used to asses the human motor intent. Furthermore, our model is non-invasive, and it does not require the placement of sensors inside muscle to measure the musculotendon unit force. The use of surface sensors significantly improves the users' comfort and widens the range of potential users.

\section{B. Impacts on Impaired Persons}

Our model makes possible several enhancements on the current state-of-the-art exoskeletons, allowing impaired people to benefit of new devices, easier to control and with a simplified training session. This positively affects the participation of impaired persons to everyday life, improving their mobility and, as a consequence, their social interaction opportunities. Furthermore, NM models allow doctors to study patients' muscle behavior without the requirement of invasive techniques. This speeds up the design of a rehabilitative therapy, which is often also more appropriate for the patient boosting his/her motor re-learning process. Decreasing the cost of the therapy and increasing its applicability and effectiveness has a large impact on impaired people as demonstrated by the data presented in the following part of this section. Impaired people can be classified in two groups. The first includes elderly individuals affected by a loss of mobility due to aging. The second includes people that are affected by motor disability due to neural or physical pathology.

1) Elderly Individuals: Our society is growing older as a direct consequence of the advances gained in the field of health science and personal health care. In Japan, for instance, the predominant age class in year 2000 was the 30 to 40 years old one while by 2050 will likely be the 70 to 80 years old one. The same data can be provided for all advanced industrial societies as United States, Canada, Australia and Europe. In Italy, the National Italian Institute of Statistics (ISTAT) is reporting a current population of persons over 75 years of age that is estimated to be comprised of 5.350 .000 individuals ( $9 \%$ of the population). This is expected to rise to over 11 millions $(19 \%)$ in 2050 . There is a high relation between the aging of the population and the number of physical disabilities. As an example, in Italy the percentage of disable people is around $5 \%$ of the population, that increases to $38 \%$ when considering only over 75 years old individuals [17]. According to current statistical projections, it is estimated that the percentage of people with some kind of disability in Europe will rise from current $11 \%$ to $18 \%$ in 2020 , mainly as a result of population aging [2].

2) Disabled Individuals: Disabilities due to neural or physical pathologies annually affect the lives of several millions of people worldwide. The most prevalent causes of disabilities are related to musculoskeletal system disorders, sensory impairments (visual and hearing), and pathologies of the nervous system (strokes, spinal cord injuries, and brain tumours). Disabilities due to stroke, for instance, annually affect 15 million people worldwide. Of these, 5 millions are left permanently paralyzed placing a burden on community [2]. 


\section{Impact on the Health Care System}

Data reported in the previous sections, clearly point out the high need for efficient solutions to assist elderly and disabled people. The number of persons requiring physical support is expected to increase in the next future. As a consequence, the health care system will need to handle a greater number of subjects bounded to spend significant amount of time in hospitals. The larger involvement of doctors and caregivers will increase, as well as the cost related to personal health care and rehabilitation [2]. Currently, labor costs for rehabilitation is roughly $60 \%$ to $70 \%$ of the U.S. expenses in the medical sector ( $\$ 30$ billion per year). If new technology could decrease, just by $5 \%$, the labor costs on only $10 \%$ of the most disabled population, the savings would be $\$ 300$ millions. A similar scenario is observable in Europe where the healthcare charges are already the $9 \%$ of the Gross Domestic Product (GDP), on average, and it is rising faster than economic growth itself, i.e. $4 \%$ a year reaching the $16 \%$ of the GDP by 2020 [2]. Providing the hospitals with intelligent robotics systems such as exoskeletons capable of taking care of the patients in an autonomous (or semi autonomous) way, will significantly decrease the number of therapists needed for a single subject and will shorten the length of stays in hospitals lowering the costs related to personal health services. Furthermore, the development of sophisticated software tool such ad NM models will help doctor to better diagnose rehabilitative treatments and decrease their cost.

\section{Impact on the Personal Health Market}

The development of personal health care solutions based on information and communication technology (e-Health) is a growing market. The global market for eHealth was estimated to be worth $€ 17.4$ billion in 2008 , increasing to over $€ 20.9$ billion by 2012, an average annual growth rate of $4.7 \%$ [2]. An increasing number of countries worldwide have already explicit eHealth policy strategies. The industry also shows an increasing interest in this field as demonstrated by exoskeletal research programs of large automobile companies, such as Honda and Toyota [6].

\section{CONCLUSiOns AND Future Work}

In this paper we proposed a NM model that can enhance the control of current state-of-the-art exoskeleton devices. This has large implications on the health care economic and social systems as the increasing number of impaired people can benefit of an enhanced interface with the devices, easier to train and control. The proposed NM model is based on the knee joint model previously developed by Lloyd et al. [1], [8], [9]. In the paper we presented a set of modifications and extensions introduced to allow the use of the model in realtime applications while preserving its prediction ability. We specifically investigated the effects of assuming the tendon infinitely stiff. Results suggested that our assumptions did not compromise the model prediction ability during gait tasks. Future work will further test the model on a broader range of movements and on more subjects. A significant improvement in terms of computation time was also achieved enabling the use of the stiff tendon model in real-time applications. We also introduced a rapid method for scaling tendon lengths to the subject's actual dimensions. This method relies on preserving the strong relationship existing between excitation and muscle activation. The suggested approach does not make any a priori assumption on muscles behavior in sharing load. This is an improvement with respect to traditional approaches. Current methods solve the force sharing problem by solving a non-linear optimization problem where the cost function assumes a certain behavior of the muscles [18]. The proposed scaling procedure will be further validated with ultrasound technology in future research.

\section{REFERENCES}

[1] D. G. Lloyd and T. F. Besier, "An EMG-driven musculoskeletal model to estimate muscle forces and knee joint moments in vivo," $J$. of Biomech., vol. 36, no. 6, pp. 765-776, 2003.

[2] "Strategic research agenda for robotics in europe," Available at: http://www.robotics-platform.eu/sra, July 2009.

[3] "A roadmap for us robotics from internet to robotics," Available at: http://www.us-robotics.us/, May 2009.

[4] E. Cavallaro, J. Rosen, J. Perry, and S. Burns, "Real-time myoprocessors for a neural controlled powered exoskeleton arm," Biomedical Engineering, IEEE Trans. on, vol. 53, no. 11, pp. 2387-2396, 2006.

[5] C. Fleischer and G. Hommel, "A human-exoskeleton interface utilizing electromyography," Robotics, IEEE Trans. on, vol. 24, no. 4, 2008.

[6] H. Herr, "Exoskeletons and orthoses: Classification, design challenges and future directions," NeuroEngineering and Rehabilitation, Journal of, vol. 6, no. 21, pp. 2387-2396, 2009.

[7] H. Kawamoto and Y. Sankai, "Comfortable power assist control method for walking aid by hal-3," in Systems, Man and Cybernetics, 2002 IEEE Intl. Conf. on, Oct. 2002.

[8] D. G. Lloyd and T. S. Buchanan, "A model of load sharing between muscles and soft tissues at the human knee during static tasks," $J$. of Biomechanical Engineering, vol. 118, pp. 367-376, 1996.

[9] T. Buchanan, D. G. Lloyd, K. Manal, and T. Besier, "Neuromusculoskeletal modeling: Estimation of muscle forces and joint moments and movements from measurements from neural command," Journal of Applied Biomechanics, vol. 20, pp. 367-395, 2004.

[10] C. Winby, D. Lloyd, and T. Kirk, "Evaluation of different analytical methods for subject-specific scaling of musculotendon parameters," $J$. of Biomech., vol. 41, no. 8, pp. 1682-1688, 2008.

[11] S. Delp, J. Loan, M. Hoy, F. Zajac, E. Topp, and J. Rosen, "An interactive graphics-based model of the lower extremity to study orthopaedic surgical procedures," Biomedical Engineering, IEEE Trans. on, vol. 37, pp. 757-767, 1990.

[12] F. E. Zajac, "Muscle and tendon: properties, models, scaling, and application to biomechanics and motor control," Crit. Rev. Biomed. Eng., vol. 17, no. 4, pp. 359-411, 1989.

[13] T. Besier, D. Sturnieks, J. Anderson, and D. Lloyd, "Repeatability of gait data using a functional hip joint centre and a mean helical knee axis," Journal of Biomechanics, vol. 36, pp. 1159-1168, 2003.

[14] M. Sartori, M. Reggiani, C. Mezzato, and E. Pagello, "A lower limb emg-driven biomechanical model for applications in rehabilitation robotics," International Conference on Advanced Robotics, In Proceedings of, vol. 24, pp. 144-158, 2009.

[15] L. Schutte, "Using musculoskeletal models to explore strategies for improving performance in electrical stimulation-inducted leg cycle ergonometry.” Ph.D. dissertation, Stanford University, 1992.

[16] J. Loan, Dymanics Pipeline. Musculographics Inc., 1992.

[17] "I disabili in Italia (Disable people in Italy)," Available at: http://www.asphi.it/DisabilitaOggi/DisabiliItalia.htm, June 2006.

[18] G. Schappacher-Tilp, P. Binding, E. Braverman, and W. Herzog, "Velocity-dependent cost function for the prediction of force sharing among synergistic muscles in a one degree of freedom model," Journal of Biomechanics, vol. 42, pp. 657-660, 2009. 